\title{
¿Es la contabilidad administrativa una herramienta útil para desarrollar la competitividad de las empresas?
}

\author{
Manuel Evia Puerto*
}

\section{Resumen}

Este trabajo pretende establecer una relación entre los antecedentes históricos de la contabilidad administrativa en el nivel internacional con el aumento de las habilidades competitivas de las organizaciones. Asimismo, ofrece algunas reflexiones acerca del presente y futuro de la misma.

Palabras clave: contabilidad administrativa, contabilidad de costos, historia de la contabilidad, gestión estratégica de costos.

\section{Introducción}

Gl entorno actual de los negocios impone a las empresas condiciones cada vez Lmás hostiles para enfrentar la creciente competencia que priva en la mayoría de las industrias. En respuesta a ese fenómeno, los administradores están obligados a plantear nuevas estrategias que les permitan conservar o incrementar su nivel de competitividad.

La mayoría de los mercados funciona bajo esquemas de competencia monopolística, en donde los participantes tienen escaso o nulo control sobre el precio de los bienes que se ofrecen, en virtud de que incrementos en el precio de venta pueden

\footnotetext{
* Profesor investigador de la Universidad del Mayab, Yucatán.Correo electrónico: manuevia@unimayab.edu.mx
} 
provocar reducción de los niveles de demanda de los consumidores, que tenderán a sustituir el producto con otro similar. Las empresas que participan en este tipo de mercados pueden optar, entre otras formas, por competir por medio de un enfoque de liderazgo en costos; esto es, manteniendo sus niveles de costos a niveles competitivos y logrando con ello incrementar sus márgenes de utilidad manteniendo sus mismos precios de mercado.

Un gran porcentaje de los administradores —en especial los que dirigen pequeñas y medianas empresas - piensa que el concepto de "reducción de costos" consiste en reducir los costos de la empresa para obtener mayores utilidades. Estos "recortes" de costos, como comúnmente se les conoce, y que normalmente se hacen de forma arbitraria, logran en la mayoría de los casos un efecto positivo de corto plazo sobre los márgenes de utilidad de la empresa, pero en el largo plazo pueden llegar a comprometer la calidad y la operatividad de la organización, puesto que se enfocan más hacia la resolución de los síntomas (efectos) de ineficiencias e ineficacias (causas) que en algunos casos son difíciles de identificar.

Por lo anteriormente expuesto, los administradores tienen la posibilidad de apoyarse en el uso de herramientas y técnicas que les permitan utilizar sus sistemas de costos como herramientas estratégicas. En el caso particular de las pequeñas y medianas empresas mexicanas, esto es un factor de importancia fundamental; por ello, es necesario investigar acerca del desarrollo y la adaptación de mecanismos y procedimientos de registro y análisis de costos que permitan a las empresas — que en muchos casos ni siquiera utilizan los sistemas de registro contable adecuados - aprovechar las ventajas competitivas de un control operativo de los costos. El factor de importancia no es conocer el costo, sino ser capaz de administrarlo; sin embargo, esto último es imposible si no se ha cuantificado adecuadamente su monto.

El autor considera que es relevante como punto de partida de estos esfuerzos hacer un recuento histórico y una evaluación del papel que la contabilidad administrativa ha detentado como herramienta auxiliar de los administradores profesionales, desde sus orígenes hasta nuestros días, así como un resumen de las principales tendencias en que pudiese evolucionar dicha técnica. 
¿Es la contabilidad administrativa una herramienta útil

para desarrollar la competitividad de las empresas?

Mucho se ha discutido acerca de lo que se entiende por "contabilidad". En nuestros días, la contabilidad se conceptualiza como un sistema de procedimientos de registro de las consecuencias económicas de las operaciones que realiza una empresa. Podemos decir que la gran mayoría de las operaciones que realiza una entidad (de cualquier tipo) son susceptibles de ser cuantificadas en términos económicos $\mathrm{y}$, por ende, originan modificaciones en la situación financiera y patrimonial de las organizaciones. Todas las transacciones que lleva a cabo un negocio que sean susceptibles de ser expresadas en unidades monetarias deberán ser objeto de registro contable.

Tradicionalmente, la contabilidad se ha conceptualizado en forma casi exclusiva como un sistema de registro en donde se conserva con detalle todas las operaciones que ha llevado a cabo el negocio; por medio de estos registros, a través de procedimientos de adición y análisis, se pueden obtener informes precisos y detallados acerca de la situación financiera y los resultados de la empresa. Por ello, se dice que la contabilidad es una herramienta para apoyar la toma de decisiones empresariales debido a que, al ser un mecanismo creado básicamente para proveer información, contribuye a despejar el nivel de incertidumbre asociado con el proceso de elección de cursos de acción.

Se acostumbra dividir, en forma primaria, a la técnica contable en dos grandes vertientes: la contabilidad financiera y la contabilidad administrativa. Ambas coexisten en el interior de las organizaciones, interactuando en forma constante.

La contabilidad financiera tiene como principal característica su sujeción a criterios normativos en cuanto a la forma en la que debe ser elaborada. Estos requerimientos son dictados por instituciones gubernamentales y por organismos profesionales, los cuales enuncian cuáles serán las normas mínimas que deberán cumplir los registros contables para asegurar sus características de calidad y comparabilidad. En México, estos criterios unificadores reciben el nombre de Principios de Contabilidad Generalmente Aceptados y son emitidos por el Instituto Mexicano de Contadores Públicos. En la actualidad, la normatividad contable mexicana está inmersa en un proceso de reestructuración, pues se están desarrollando e implementando en la actualidad otras normas contables, denominadas Normas de Información Financiera, promulgadas por el Consejo Mexicano para la Investigación y Desarrollo de Normas de Información Financiera y que vendrán a sustituir en un futuro cercano a los citados principios de contabilidad. 
En contraposición, la contabilidad administrativa no está sujeta, al menos en México, a ningún tipo de normatividad; en algunos países como Francia sí lo está (Lebas, 1994). Es por ello que los sistemas de contabilidad administrativa pueden ser creados a la medida de las organizaciones, respondiendo a las demandas específicas de cada una en particular.

Puede decirse que el objetivo final de la contabilidad financiera es proporcionar una visión integral de las modificaciones sufridas en el volumen y composición del patrimonio de una entidad a lo largo de un determinado periodo, como consecuencia de las transacciones que la misma lleve a cabo y de los imponderables a los que esté sujeta. De esta forma, presenta los resultados de una entidad como un todo y sujeta a determinados criterios normativos. Por su parte, la contabilidad administrativa permite evaluar funciones aisladas del negocio y los resultados de determinadas acciones particulares emprendidas por la empresa. Contempla, asimismo, la predicción de los resultados contables y financieros de la entidad. Existe la idea de que la contabilidad administrativa es la relacionada con los costos de la empresa, estableciéndose un símil entre la misma y la contabilidad de costos. La contabilidad de costos es la técnica para determinar el costo de un proyecto, de un proceso o de un producto; este costo se determina a través de una medición directa, de una asignación arbitraria o de una asignación sistemática o racional. Se puede decir que la contabilidad de costos es una parte integral de la contabilidad administrativa, por lo que los costos de producción y venta no representan en forma exclusiva el ámbito de estudio de la segunda. La contabilidad de costos ha creado un puente entre la contabilidad administrativa y la contabilidad financiera. La contabilidad de costos se integra con la contabilidad financiera mediante el suministro de una parte de la información cuantitativa de los costos básicos que los administradores necesitan para desempeñar sus tareas.

\section{El pasado de la contabilidad administrativa}

\section{Orígenes}

La contabilidad administrativa surge en el momento en que los empresarios se percatan de que la situación financiera de la organización no puede ni debe darse como respuesta ante los cambios en el entorno. En el siglo XIX, las empresas se 
¿Es la contabilidad administrativa una herramienta útil

para desarrollar la competitividad de las empresas?

administraban mediante un concepto reactivo: los cambios en la producción, en los niveles de precios y otras variables de la empresa se decidían como respuesta a los cambios en el medio ambiente del negocio. La contabilidad administrativa surge cuando los empresarios deciden actuar internamente en sus organizaciones para dirigirlas tanto financiera como operativamente (Duarte, 2001).

En la actualidad, un porcentaje de las empresas desarrolla planes de acción expresados en términos monetarios conocidos como presupuestos. Éstos contemplan la creación, implementación y desarrollo de las estrategias que la empresa debe llevar a cabo para lograr la consecución de sus objetivos. En la antigüedad, las organizaciones no llevaban a cabo procesos formales de planeación. El objetivo primordial era el de incrementar la producción para no perder ventas. Se daba escasa atención a los costos, los cuales se calculaban de forma muy simple, y al uso eficiente de recursos. Durante la Revolución Industrial los empresarios se percataron de los beneficios que podían obtener mediante la reducción de costos y el incremento en la eficiencia de sus procesos. Estos empresarios descubrieron la utilización del concepto de economías de escala, el cual significa la obtención de reducciones en los costos de producción cuando un producto se fabrica en masa (Milgrom y Roberts, 1992).

\section{Siglo XIX}

Dentro de los antecedentes modernos de la contabilidad administrativa figura el sistema de costos establecido en Lyman Mill, un molino textil de Nueva Inglaterra, que en 1855 permitió a los directivos de la empresa controlar la eficiencia de los costos de conversión, con base en libros de contabilidad por partida doble y que arrojaba información acerca del costo de los productos terminados, de la productividad de trabajadores, del impacto de los cambios de la distribución de la planta, así como controles de recepción y uso del algodón (Baujín, Vega, Armenteros, Frías y Pérez, 2003).

El costo de producción se calculaba adicionando únicamente el costo de la mano de obra a la materia prima utilizada. Se puede encontrar un antecedente interesante en el sistema de costos implementado en 1865 por Andrew Carnegie —a quien se le considera el introductor de la contabilidad de costos al mundo empresarial - en su compañía Carnegie Steel Company. El sistema que diseñó consistió en la 
recolección de datos de todos los costos que tenían relación con los procesos de producción. Al final, permitía evaluar el desempeño de cada departamento. Estos controles sobre los costos permitieron a la empresa responder de manera más oportuna a los cambios en los precios del acero, proporcionándole una ventaja sobre sus competidores (Johnson y Kaplan, 1987). Otra industria que contribuyó en forma importante al desarrollo de la contabilidad de costos fue la ferroviaria, que presentaba el reto de calcular el costo de un servicio, independientemente de las diferentes distancias recorridas. En esa industria se generó el concepto de cuota por unidad de costo, al determinarse una cantidad de costo por cada tonelada transportada. La preocupación central de los empresarios del siglo XIX era conocer su costo de producción. La creciente complejidad en los sistemas de producción derivó en una necesidad de contar con más y mejor información financiera. A esta etapa se le conoce como "etapa de la administración científica".

De 1889 a 1895 Fredrick Taylor, uno de los pioneros del control de administración industrial, desarrolló la contabilidad analítica, la asignación de costos indirectos, el cronometraje y el seguimiento de los tiempos de mano de obra directa, los estándares, la asignación de costos indirectos mediante los tiempos de mano de obra o de los tiempos máquina, la gestión de la existencia y la remuneración al rendimiento. Entre los años de 1890 y 1915 se llevó a cabo un proceso de diseño de la estructura básica de la contabilidad de costos mediante la introducción de conceptos como el establecimiento de procedimientos de distribución de los costos indirectos de fabricación, valuación de inventarios, estimación de elementos de costo y contabilización de activos a valor de adquisición (Baujín et al., 2003).

Durante la penúltima década del siglo XIX, los ingenieros de la industria metalmecánica se dieron a la tarea de diseñar un sistema de contabilidad que tenía como objetivo primordial el cálculo de los materiales y la mano de obra requeridos durante un proceso de producción (antes de que ese proceso iniciara). Esto es lo que posteriormente se denominó como "costos estándares". Para poder fijar esos costos predeterminados, se hacía indispensable el contar con información histórica que, en forma de estadísticas, permitiera pronosticar el comportamiento de los costos futuros. 
¿Es la contabilidad administrativa una herramienta útil para desarrollar la competitividad de las empresas?

\section{Siglo XX: 1900-1930}

Entre 1915 y 1920, Alexander Hamilton Church planteó la necesidad de controlar los costos indirectos a través de todo su recorrido por la empresa procediéndose finalmente a su asignación en función de referencias distintas a los costos directos (Baujín et al., 2003). Church enunció que se deben relacionar los costos de todos los productos fabricados por la empresa con la suma de las utilidades obtenidas como consecuencia de las ventas de cada producto. Una limitante importante a las aportaciones de este investigador la constituyó el hecho de haber omitido la utilización de los gastos indirectos de fabricación (Johnson y Kaplan, 1987).

Con el advenimiento del siglo XX, las empresas mostraron una propensión a volverse generalistas en vez de especializarse en producir un solo artículo. No es sino hasta los primeros años del siglo pasado que los empresarios se percataron de los beneficios que se derivaban de producir sus propias materias primas o, bien, de adquirir las empresas de sus proveedores; a este fenómeno se le conoce como integración vertical. Adicionalmente, es en ese periodo donde se presenta la creación de canales de distribución para los productos que manufacturaban las empresas (Duarte, 2001).

Estas acciones permitieron a las empresas incrementar sus volúmenes de utilidades, de tal forma que en 1907, y como resultado de un largo proceso de innovaciones iniciado en 1902, Donaldson Brown, colaborador de Pierre Du Pont

Rendimiento sobre la Inversión $=\frac{\text { Utilidad de operación }}{\text { Promedio de activos de operación }} \quad$ Fórmula 1

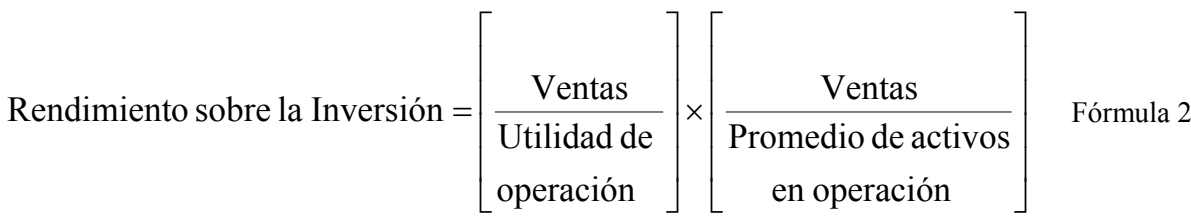

Rendimiento sobre la Inversión $=\left[\begin{array}{c}\text { Margen de utilidad } \\ \text { en operación }\end{array}\right] \times\left[\begin{array}{c}\text { Rotación de activos } \\ \text { en operación }\end{array}\right]$

Fórmula 3 
de Nemours, inventó una fórmula que relaciona el índice de rentabilidad del capital con el índice de rotación de dicho capital. Así, creó un sistema presupuestal que incorpora la medida de desempeño conocida como Return Over Investment (ROI) o Rendimiento sobre la Inversión (RSI) (DuPont, 2005).

Según Johnson y Kaplan (1987), a efectos de poder llevar a cabo el cálculo del RSI, es necesario realizar el registro contable de la totalidad de los activos de la empresa. La empresa Du Pont registraba el monto de sus activos fijos en una cuenta que llamaba "inversiones permanentes". Esta cuenta proporcionaba la información que era necesaria para percatarse del monto de los flujos de efectivo requeridos para llevar a cabo nuevos proyectos de inversión y que era generada a través de un pronóstico de los flujos de efectivo futuros que a la larga fueron utilizados como medida del desempeño de la empresa debido a que al concluir el periodo se realizaba una comparación entre los flujos estimados a priori con los flujos reales y tomando como base esta comparación se analizaban las desviaciones entre los estándares y los flujos reales.

Esta aportación de Brown a la ciencia económica constituyó la base de las finanzas modernas (Duarte, 2001). El modelo de valuación de activos y de presupuestación de capital utilizado en la actualidad requiere descontar al valor presente de los flujos de inversión futuros de un activo el monto de la inversión asociada al mismo el día de hoy. De igual forma, la compañía Du Pont creó métodos de control para sus procesos fabriles; además promovió la elaboración de dos reportes mensuales: uno que registraba todas las actividades de producción relacionadas con un artículo y otro que registraba los precios y los volúmenes de venta. La información de ambos reportes se comparaba y se obtenían las utilidades (Atkinson, Banker, Kaplan y Young, 1997).

Cerca de 1920, Brown dejó la compañía Du Pont para convertirse en el director de Finanzas de General Motors, bajo la dirección de Alfred Sloan. Bajo el liderazgo de ambos, esta empresa desarrolló muchas herramientas de contabilidad administrativa que tenían como finalidad cumplir con la filosofía operativa de "control centralizado con responsabilidad descentralizada" (General Motors, 2005). El término "responsabilidad descentralizada" se refiere a la autoridad que los administradores de divisiones locales tienen para tomar sus propias decisiones sin tener que buscar una autorización superior en cuestiones de precio, mezcla de productos, relaciones con los clientes, diseño de productos, adquisición de 
¿Es la contabilidad administrativa una herramienta útil

para desarrollar la competitividad de las empresas?

materiales y procesos operativos apropiados. Esto permite a los administradores la toma de decisiones oportunas que permiten aprovechar oportunidades repentinas. El control descentralizado para las operaciones implicaba que la información relativa a su rentabilidad divisional y a la operación llegara en forma periódica a los administradores locales. Estos reportes permitían a la alta dirección corroborar si los esfuerzos administrativos de los mandos operativos se apegaban a la consecución de las metas de la organización.

Algunos de los elementos que contenían los sistemas de contabilidad administrativa en General Motors eran:

1. Un proceso de presupuestación anual. Este proceso determinaba si los planes de cada división eran consistentes con los planes de otras divisiones, $v . g$. que la división de ventas no espere vender más de lo que la división de manufactura espera producir. De igual forma, este proceso de presupuestación identificaba cómo los flujos de fondos serían generados y subsecuentemente cómo serían asignados a cada división. Además, este proceso promueve la coordinación entre las metas de la alta gerencia con los objetivos divisionales.

2. Reportes semanales de ventas y presupuestos flexibles mensuales. Los reportes de ventas tenían la finalidad de monitorear el desempeño de las ventas reales contra las ventas presupuestadas. Los presupuestos flexibles proveen estimaciones acerca de cuál debe ser el nivel de gasto y la mezcla de producción y ventas en el periodo más reciente. El sistema de proveer reportes de ventas semanales y presupuestos flexibles proporciona retroalimentación oportuna a los gerentes divisionales acerca de las operaciones actuales, de tal forma que pueden tomar acciones para ajustarse rápidamente a los cambios en el entorno operativo de la organización.

3. Reportes de desempeño anuales incluyendo rendimiento sobre la inversión. El reporte de desempeño anual proporcionaba a la administración central información que le permitía asignar recursos y esfuerzos administrativos a las diferentes divisiones de negocio utilizando criterios uniformes, tales como el RSI. Sloan apreció en gran medida la utilidad del RSI como 
medida de evaluación, como puede apreciarse en la sentencia siguiente: "Ningún otro principio que haya conocido sirve mejor que la tasa de rendimiento como una ayuda objetiva para evaluar negocios" (Giroux, S.F.).

El sistema de contabilidad administrativa de General Motors permitía a una organización compleja planear, coordinar, controlar y evaluar las operaciones de divisiones operativas independientes como Chevrolet y Pontiac; también permitió a los administradores de esas divisiones perseguir tenazmente sus objetivos divisionales en tanto que se mantienen coherentes con las metas corporativas (Atkinson et al., 1997).

Para 1930, Du Pont y General Motors habían logrado desarrollar sistemas de contabilidad administrativa que de forma muy competente daban resultados a las administraciones de las organizaciones. El autor considera que ese "liderazgo temprano" fue un factor determinante para posicionar a ambas empresas en la situación que detentan en la actualidad.

Los años posteriores a 1930 se caracterizaron por el creciente interés mostrado por los empresarios en conocer sus costos de producción. En aquellos tiempos en que los sistemas de procesamiento de datos eran muy rudimentarios, resultaba sumamente costoso el procesamiento de datos relativos a los costos de múltiples productos o líneas de productos. A raíz de esto es que la contabilidad administrativa comenzó a ser evaluada mediante el enfoque de "costo-beneficio". En esas fechas también comenzó a generalizarse entre los empresarios la idea de tender a "profesionalizar" la administración de sus negocios, contratando administradores profesionales para dirigirlos. Esto condujo a la implementación de controles tales como la auditoria a la información financiera para evaluar el desempeño de los administradores.

La mencionada complejidad en los procesos de costeo redundó en la búsqueda de medios más simples para calcular los costos. Ello condujo al desarrollo de los métodos de valuación de inventarios, los cuales se ocupan de controlar el costo de las materias primas. Se estaba dejando a un lado la incorporación de los Costos Indirectos de Fabricación (CIF) (Johnson y Kaplan, 1987). Debido a las necesidades de información, en las primeras décadas del siglo XX todos los costos eran asignados como costos directos de producción, siguiendo cada actividad desarrollada para producir un artículo; sin embargo, hay costos que no 
¿Es la contabilidad administrativa una herramienta útil

para desarrollar la competitividad de las empresas?

se pueden asociar directamente a la fabricación de un producto. Esto fue reconocido por los administradores, quienes empezaron a asignar los CIF utilizando diferentes métodos de prorrateo, tales como las horas de mano de obra utilizada o los metros cuadrados de planta. Durante la década de 1940, las escuelas de negocios enseñaban a los estudiantes las técnicas de valuación de inventario como la única actividad desempeñada por la contabilidad de costos (Johnson y Kaplan, 1987).

\section{Sistemas alternativos utilizados en otras naciones}

Después de finalizar la Segunda Guerra Mundial, se gesta un proceso de reconstrucción en los países de Asia y Europa. Los sistemas industriales de estos continentes son diferentes al modelo norteamericano, en parte por cuestiones culturales y en parte debido a situaciones económicas. En algunos países, como Alemania y Japón, que se encontraban en ruinas y ocupados por los aliados, el proceso de reconstrucción industrial fue rápido (Giroux, S.F.).

¿Cuál fue el factor que provocó que Japón, cuya economía creció a una tasa del $10 \%$ hasta cerca de 1990, se convirtiese en pocos años en la segunda economía más grande del planeta? Según Giroux, los japoneses utilizaron el método llamado ahora Competitive Benchmarking (CB), copiando tecnología e ideas de compañías líderes en el nivel mundial. Uno de los más respetados consultores en este proceso fue Edwards Deming, matemático norteamericano considerado como el creador y principal impulsor del enfoque administrativo de la Administración de la Calidad Total (ACT).

Una de las empresas japonesas más exitosas es Toyota Motor Company, fabricante de automóviles. Los directivos de la compañía Taichii Ohno y Shigeo Shingo empezaron a incorporar los sistemas de control de la producción desarrollados por Charles E. Sorensen en la Ford Motor Company, adicionándolos con controles estadísticos de la producción y otras técnicas dentro de un enfoque denominado "Sistema de producción Toyota" o "Sistema de manufactura Justo a Tiempo" (JAT). En él se reconocía el rol central del control del inventario. Este desarrollo tomó lugar entre 1949 y 1975, extendiéndose rápidamente a otras empresas japonesas. Cuando las ganancias en productividad 
y calidad se hicieron evidentes, ejecutivos de diversos países viajaron a Japón para investigar acerca de ello. A su retorno, empezaron a importar conceptos superficiales, tales como los círculos de calidad (Strategos Inc, S.F.).

\section{El estancamiento: 1950-1980}

Según Johnson y Kaplan en su obra Relevance Lost (1987), las empresas norteamericanas fueron líderes mundiales desde los inicios del siglo XX hasta la década de los setenta. En los setenta y en los ochenta, la administración de la producción norteamericana y la contabilidad de costos se volvieron obsoletas. Las empresas enfrentaban altos costos de producción y pérdidas importantes en la calidad. Antes de 1950, los administradores en los Estados Unidos utilizaban la información de la contabilidad de costos, principalmente, para propósitos de planeación, mientras que el control operativo se basaba en información ajena a los registros contables. Desde 1950, se presentó la tendencia de que los administradores se enfocasen en los resultados contables para efectos de control. Después de la posguerra se hizo evidente la necesidad de dar "variedad" a la producción (así lo demandaban los consumidores que querían productos que satisficieran sus necesidades personales). Se necesitó de gran capacidad productiva para alcanzar economías de escala, pero sin provocar una expansión desmedida en los niveles de inventarios. Los CIF se incrementaron, aumentando su importancia y resultando en problemas de asignación de costos. La solución percibida fue incrementar el volumen de producción para prorratear el costo fijo entre más unidades y con ello reducir el costo unitario.

Johnson y Kaplan hacen una severa crítica de la contabilidad administrativa norteamericana de la posguerra, pues la industria norteamericana no era competitiva para enfrentar los costos de producción menores y la mayor calidad de los productos provenientes de Asia y Europa. Las industrias norteamericanas en la década de los ochenta eran prácticamente incapaces de producir artículos de alta calidad porque los competidores foráneos eran capaces de dominar su mercado y de conducir a las empresas norteamericanas al fracaso. En la crítica de Johnson y Kaplan, la contabilidad administrativa fue parte del problema. 
¿Es la contabilidad administrativa una herramienta útil

para desarrollar la competitividad de las empresas?

De acuerdo con estos autores, el esquema tradicional de contabilidad de costos desarrolló en los administradores "una mentalidad de contabilidad financiera", en la cual el enfoque en el inventario y el costo de ventas era más importante que la información precisa de los costos de producción. Las utilidades de corto plazo eran cruciales, pero a largo plazo no existía un enfoque en los clientes, empleados y proveedores. A principios del siglo XX, la mano de obra directa y los materiales directos representaban en promedio el $90 \%$ del costo de producción, por lo que prorratear los CIF con base en la mano de obra directa parecía razonable. Al final del mismo siglo, la mano de obra directa representaba solo el $10 \%$ del costo total, mientras que los CIF ascendían en promedio al $60 \%$, de tal forma que asignar los CIF tomando como base las horas de mano de obra carecía de sentido. Adicionalmente, no era común considerar el control de calidad total o la meta de cero defectos (prácticas comunes en el Japón). Hay una anécdota a este respecto que conviene mencionar y resulta sumamente curiosa. IBM, una empresa con niveles aceptables de calidad, firmó un acuerdo para importar a los Estados Unidos componentes para computadoras fabricados en Japón. Dentro del clausulado del contrato mencionaban que era aceptable un límite de tres componentes defectuosos por cada 10,000 correctos. Cuando se recibió el primer embarque venía acompañado con una nota que decía:

[...] nosotros los japoneses pasamos largo rato tratando de entender las prácticas comerciales en Norteamérica. De todas maneras los tres componentes defectuosos por cada 10,000 en buen estado han sido incluidos y están empacados por separado. Esperamos que esto les agrade. (Duarte, 2001: 65)

Sin embargo, durante los periodos de calma de finales de la década de los ochenta, los Estados Unidos fueron los líderes mundiales en productividad. Muchas empresas eran exportadores netos, pero los problemas eran reales y severos: aproximadamente el $70 \%$ de las manufacturas norteamericanas enfrentaban competencia extranjera directa; existía un déficit comercial sustancial en acero, automóviles, textiles y electrónica. Las empresas norteamericanas fueron completamente desplazadas en algunas industrias; la electrónica fue el ejemplo más severo (Giroux, S.F.). 


\section{La respuesta norteamericana: de 1990 a nuestros días}

A finales de la década de los ochenta, los consumidores percibían a los productos japoneses y de otros países como de mayor calidad comparados con los productos norteamericanos. Las empresas de los Estados Unidos perdían tanto en mercados internos como externos; por ejemplo, la industria automotriz registró pérdidas millonarias: en 1987 el 30\% de las ventas de automóviles en los Estados Unidos correspondieron a vehículos importados y la industria registraba un déficit de 60 billones de dólares; para 1990 las compañías automotrices recuperaron $75 \%$ del mercado norteamericano y lograron niveles aceptables de rentabilidad. La industria adoptó prácticas industriales japonesas como el JAT y la ACT, lo cual produjo buenos resultados.

Goldratt (citado en CPA finder, S.F.) apunta que "la contabilidad de costos es el enemigo número uno de la productividad"; según Jayson (citado en CPA finder, S.F.) Goldratt posteriormente suavizó su postura argumentando que "el costo más que la contabilidad es el culpable"; sin embargo, Goldratt sostuvo que las medidas de costo en uso en la actualidad "envían señales equivocadas a los administradores, quienes están tratando de controlar inventarios y gastos de operación" (Cheatman, 1983, citado en CPA finder, S.F.).

De acuerdo con Johnson (citado en Giroux, S.F.), los componentes principales para que una empresa pueda lograr competitividad internacional incluyen relaciones de largo plazo con los consumidores, flexibilidad en los procesos (dotar a los trabajadores de capacidad para tomar decisiones o empowerment), la eliminación de restricciones y enfocarse nuevamente en la información contable, que no proveía información acerca de los consumidores o de la calidad del producto; más que controlar operaciones, la información contable provee información valiosa, desempeñando un rol de soporte.

La "respuesta norteamericana" ha funcionado bien desde la década de los noventa, haciendo un mayor énfasis en la calidad, la satisfacción del consumidor y el empowerment a los empleados (a partir de ahora "incremento del potencial"). Según Giroux (S.F), la contabilidad administrativa ha desempeñado un papel trascendental en el éxito global de la industria norteamericana en los últimos años. 
¿Es la contabilidad administrativa una herramienta útil

para desarrollar la competitividad de las empresas?

\section{Situación actual de la contabilidad administrativa}

De acuerdo con Johnson y Kaplan (1987), la teoría tradicional de la contabilidad de costos no reflejaba en forma alguna el mundo real de mediados de la década de los ochenta, el cual se caracterizaba por fenómenos como complejos sistemas de manufactura, alta tecnología y automatización, muy alto nivel de CIF y cambios en el trabajo de la organización. Esos autores argumentaron la necesidad de incluir nuevas medidas de costo en un determinado número de áreas incluyendo:

a. Nuevas medidas de desempeño para el control de procesos.

b. Costeo basado en actividades para productos o servicios.

c. Necesidad de costear la cadena de valor en su totalidad.

d. Establecer mejores medidas para la evaluación del desempeño, incluyendo medidas no financieras.

Según Cotton (2005), Tom Johson publicó en 1992 una secuela del libro Relevance Lost (en el que exponía la problemática que enfrentaba la contabilidad de costos) intitulada Relevance Regained; en esta obra, se argumenta que los sistemas efectivos de control administrativo deben reenfocarse de "control de arriba para abajo" a "incremento de potencial de abajo para arriba"; señala que es vital para las empresas contar con sistemas de información que liguen acciones con los mandatos de la competencia global; también menciona que los equipos de trabajo autoadministrados deben poseer información necesaria para resolver problemas de exceso de variaciones, retrasos y complejidad en los procesos.

En abril de 1986 se llevó a cabo un simposio en los Estados Unidos de Norteamérica con el tópico de "Contabilidad Administrativa para los Noventas", organizado por el Instituto de Contadores Administrativos, la Escuela de Negocios de la Universidad de Harvard y la Asociación Americana de Contabilidad. Este simposio se recuerda como un parteaguas en el desarrollo de la contabilidad administrativa (Cotton, 2005), pues se presentaron innovaciones importantes al estado del arte de la contabilidad administrativa, varias de ellas promulgadas bajo el enfoque de la "Gestión Estratégica de Costos" (GEC). Estas innovaciones se enlistan en la bibliografía especializada y se practican en forma exitosa en varias organizaciones. Entre ellas pueden destacarse: 
a. Costeo basado en actividades (CBA) y administración basada en actividades (ABA).

b. Medidas de capacidad y administración.

c. Análisis de la rentabilidad del cliente.

d. Valor Económico Agregado (VEA).

e. Sistemas de recursos de planeación de empresas.

f. Sistema de Costeo Justo a Tiempo (JAT).

g. Medidas de desempeño no financieras y el "Tablero de comando" (Balanced Scorecard).

h. Sistemas de calidad y administración del tiempo, incluyendo la "Teoría de las Restricciones".

i. Asignación de costos y precios objetivo.

Esta lista, tomada de Cotton (2005), no pretende enunciar todos los nuevos descubrimientos que se han incorporado recientemente a la teoría de la contabilidad administrativa; solamente se enumeran los que han tenido mayor grado de aceptación entre las organizaciones.

¿Qué técnica de contabilidad administrativa debe ser aplicada para cada caso en particular? Sharma (2000) llevó a cabo una investigación en la que se planteó las siguientes preguntas:

1. ¿Afecta el tamaño de las organizaciones los beneficios relativos de las prácticas de contabilidad administrativa tradicionales y emergentes?

2. ¿Afecta el tipo de industria (manufactura o no manufactura) los beneficios relativos de las prácticas de contabilidad tradicionales y emergentes?

3. ¿Afecta la estrategia de la organización (precio, diferenciación o ambos) los beneficios relativos de las prácticas de contabilidad tradicionales y emergentes?

La investigación realiza una clasificación de las técnicas de contabilidad administrativa en técnicas "tradicionales" y técnicas "emergentes", tal como se muestra en la tabla siguiente. 
¿Es la contabilidad administrativa una herramienta útil para desarrollar la competitividad de las empresas?

\section{Tabla 1}

Prácticas tradicionales y emergentes de contabilidad administrativa

\begin{tabular}{|l|l|}
\hline \multicolumn{1}{|c|}{ Prácticas tradicionales } & Prácticas emergentes \\
\hline Presupuestación & Medidas de desempeño balanceado \\
\hline Análisis de productos & Planeación estratégica \\
\hline Métodos de costeo & Medidas basadas en los empleados \\
\hline & Benchmarking \\
\hline & Técnicas basadas en actividades (CBA y ABA) \\
\hline & Métodos de creación de valor \\
\hline
\end{tabular}

Fuente: Sharma, 2000

Para contestar las preguntas anteriores, se envió un cuestionario a una muestra de 1,500 profesionales de la contabilidad seleccionados en forma aleatoria. Se obtuvo una tasa de respuesta del $22 \%$. En forma breve se pueden contestar las preguntas de investigación como sigue:

Con respecto a la pregunta 1, se consideró que las prácticas emergentes eran significativamente más benéficas para las empresas medianas y grandes. Las técnicas emergentes de métodos de creación de valor y técnicas basadas en actividades (CBA y ABA) fueron consideradas las más benéficas. Las técnicas basadas en actividades y los métodos de creación de valor se perciben como más benéficas a medida que el tamaño de la firma se incrementa. Los beneficios de las técnicas de planeación estratégica incrementaron significativamente entre las organizaciones pequeñas y medianas.

En respuesta a la pregunta 2, las prácticas tradicionales en empresas no manufactureras fueron percibidas con bajo potencial para crear beneficios. Consideramos que esto se explica debido a que las prácticas tradicionales se enfocan de forma preponderante a empresas fabriles. Las prácticas emergentes se percibieron como benéficas para cualquier tipo de empresa. 
Con respecto a la pregunta 3 , se encontró que las estrategias de precio y diferenciación no interactúan de forma significativa una con otra para afectar los beneficios producidos por las prácticas tradicionales y/o las emergentes.

El citado estudio concluye que "las organizaciones en el nuevo milenio requerirán adoptar un enfoque más holístico a la administración" (Sharma, 2000). Los administradores requerirán tanto de los métodos tradicionales de contabilidad administrativa como de los emergentes para tomar mejores decisiones. Factores clave como tamaño, industria y prioridades estratégicas tienen impactos diferentes en las prácticas administrativas: el tamaño es significativo para las prácticas emergentes, mientras que el tipo de industria lo es para las prácticas tradicionales; adicionalmente, las prioridades estratégicas también afectan dramáticamente los beneficios de las prácticas tradicionales y emergentes (Sharma, 2000).

\section{Futuro de la contabilidad administrativa}

Tal como se mencionó anteriormente, el papel de la contabilidad administrativa es el de proporcionar soporte a la toma de decisiones de la empresa, ésa es su razón de ser; su utilidad se verá demostrada en la medida en que se logre su principal objetivo.

La contabilidad administrativa se enfoca básicamente al futuro de la organización; por ello, es indispensable establecer la relación de la contabilidad administrativa con el proceso administrativo. La primera es una herramienta sumamente útil para las fases de planeación y control del mencionado proceso: en la etapa de planeación, por medio de la utilización de sistemas de planeación y control de utilidades (presupuestos); en la fase de control a través de sistemas de medición del desempeño como el "Tablero de control".

Por lo anterior, consideramos que la contabilidad administrativa conservará e incrementará en el futuro su grado de relevancia como elemento fundamental en la administración organizacional, pero no únicamente como un sistema de registro transaccional, sino como un mecanismo de control de la administración estratégica. Los contadores administrativos deben estar preparados para hacer frente a este cambio en el paradigma en el marco teórico de la disciplina. Un factor de 
¿Es la contabilidad administrativa una herramienta útil

para desarrollar la competitividad de las empresas?

suma importancia es la necesidad de reorientar los planes de estudio de las instituciones educativas en materia de contabilidad administrativa, reforzando la enseñanza de las nuevas tecnologías disponibles en la materia. Asimismo, el contador administrativo debe estar muy familiarizado con los avances que en materia de tecnología de información se presenten en el área. Sangster (1994) dice que se requiere de un esfuerzo educativo importante para concientizar a los contadores administrativos de los beneficios que les reportarán los sistemas de cómputo, tanto para llevar a cabo su labor como para auxiliarlos en el proceso de toma de decisiones administrativas.

Kaye (2001) enuncia que el contador administrativo está tomando un rol emergente en la formulación, desarrollo e implementación de estrategias de información al interior de las organizaciones; en su opinión, los contadores administrativos se han adaptado a la tecnología de la información y han estado activos en la explotación de sistemas de procesamiento de datos desde la introducción de los mismos. Las habilidades administrativas, aunadas al conocimiento de los sistemas de información, formarán poderosas herramientas que permitirán la participación del contador administrativo en el desarrollo de estrategias de información. El desarrollo de una estrategia de información involucra la integración de tecnología, contenido informativo y actividad administrativa.

Gary Cokins, autor de Activity Based Cost Management, An Executive's Guide, comentó en una entrevista: "La contabilidad administrativa evolucionará hacia una forma de economía administrativa. Los administradores del siglo XXI requerirán más integración con sus organizaciones. Debido a la introducción de tecnologías que proporcionan información basada en actividades, el paso al cambio se ha dado" (SmartPros Editorial Staff, S.F.).

\section{Conclusiones}

La contabilidad administrativa ha sido desde sus orígenes una herramienta sumamente útil a la administración de las organizaciones. Desde nuestro punto de vista, las compañías que en su momento desarrollaron las técnicas de contabilidad administrativa descritas a lo largo de este trabajo obtuvieron una ventaja competitiva originada por la aplicación de las mismas, la cual contribuyó a su posicionamiento como líderes de mercado. Sin embargo, durante el proceso 
evolutivo de la contabilidad administrativa, ésta no ha podido adecuarse a la evolución del entorno de negocios; no obstante, las nuevas tendencias de costeo, donde se evidencia el uso de los costos como herramienta estratégica, la han vuelto a situar en un papel de total relevancia como una valiosa ayuda de las funciones de gestión y control.

Consideramos que el futuro de la contabilidad administrativa está íntimamente ligado a las ciencias computacionales. El desarrollo de paquetería de cómputo especializada que pueda ser comercializada en condiciones aceptables para las empresas pequeñas y medianas contribuiría a fomentar la aplicación de los sistemas de contabilidad administrativa en las organizaciones, así como la implantación de procedimientos de capacitación que den a conocer las nuevas técnicas de costeo y los beneficios que podrían reportar a los administradores de las empresas. Asimismo, es necesario realizar investigaciones orientadas a desarrollar nuevos enfoques de administración de costos que respondan a las necesidades específicas de las pequeñas y medianas empresas mexicanas.

\section{Bibliografía}

ATKINSON, A.A., et al. (1998), Management Accounting, 2a. ed., Prentice Hall, EUA.

BAUJíN, P., et al., Evolución histórica de la contabilidad de gestión, en http://www.gestiopolis.com/recursos4/docs/fin/evocontabi.htm, 7 de junio de 2005.

COTTON, B. (abril, 2005), "Relevance Redux"- Management Accounting Today, Chartered Accountants Journal, 6-12.

CPA Finder "Cost Accountants and Cost Accounting", sitio web: http://www.cpafinder.com/accounting/cost-accountant-cost-accounting.html, 7 de junio de 2005 (de CPA Zinder). 
¿Es la contabilidad administrativa una herramienta útil

para desarrollar la competitividad de las empresas?

DUARTE OLVERA, F. (2001), Administración estratégica de costos como base para la obtención de ventajas competitivas, Instituto Mexicano de Contadores Públicos, México.

DU PONT, Donaldson Brown, sitio web:

http://heritage.dupont.com/floater/fl_brown/floater.shtml 8 de junio de 2005 (de Du Pont de Nemours and Company).

GENERAL MOTORS COMPANY, "The Company History. General Motors Company", http://www.gm.com/company/corp_info/history/gmhis1920.html, 6 de junio de 2005.

GIROUX, G. (S.F.), American Big Business and Cost Accounting, http://acct.tamu.edu/giroux/Americanbigbusiness.html, 7 de junio de 2005.

JOHNSON, T y R. Kaplan (1995), Relevance Lost, Boston, Harvard Bussiness School Press.

"Just in Time, Toyota Production \& Lean Manufacturing: Origins and History of Lean Manufacturing", sitio web: www.strategosinc.com, 8 de junio de 2005 (de Strategos Consultants Enginners Strategists).

KAYE, Ronald (2001), "The Role of Management Accountants in Information Strategy", Journal of Information Technology, pp. 251-264.

LEBAS, Michel (1994), "Managerial Accounting in France: Overview of Past Tradition and Current Práctice", European Accounting Review, pp. 1-20.

MILGROM, P. y J. Roberts (1992), Economics Organizantion and Management, Prentice Hall, EUA.

SANGSTER, A. (1994), "The Adoption of IT Management Accounting: The Expert Systems Experience", Journal of Information Technology, pp. 159-169. 
SHARMA, R. (2000), "From Relevance Lost to Relevance Regained: Management Practice in the New Millenium", International Federation of Accountants, http://www.ifac.org/Library/SpeechArticle.tmpl?NID=9762908175453, 6 de junio de 2005 .

SMART PROS, "Expert Describes Future of Managerial Accounting", sitio web: http://finance.pro2net.com/x34475.xml, 8 de junio de 2005 (de Smart Pros Editorial Staff). 